\title{
Struggling with Gift-Giving Obligations: When Mobile Messages are too Laborious to Reciprocate
}

\author{
Antti Salovaara \\ Helsinki Institute for Information Technology (HIIT) \\ Helsinki University of Technology and University of Helsinki \\ P.O. Box 9800, 02015 TKK, Finland \\ antti.salovaara@ hiit.fi
}

\begin{abstract}
Messaging with new media should be fun, but sometimes participating in conversations can become a burden for users due to the effort required. In this paper, examples of such situations are presented from a study on a multimedia program for mobile phones that can be used to create MMS-based comic strips. The examples show how the social system of gift-giving obligations, originally presented by anthropologist Marcel Mauss, still has a role in everyday social interaction of today. The identified problems give rise to a number of design implications to alleviate problems in gift-giving.
\end{abstract}

\section{Categories and Subject Descriptors}

H5.3 [Information Interfaces and Presentation (e.g., HCI)]: Group and Organization Interfaces - computer-supported cooperative work.

\section{General Terms}

Design, Human Factors.

\section{Keywords}

Gift-giving, non-appropriation, mobile multimedia, messaging

\section{INTRODUCTION}

One of the essential requirements for successful communication in social interaction is that people attend to what others say, and display this in an appropriate manner $[2,6]$. Through this ongoing interactional process, by acknowledging the expressions of the other and contributing to the discussion, the parties are able to maintain a sense of mutual alignment and respect. If the process breaks down and is not corrected in an accountable way, the exchange of ideas rapidly comes to a halt. This happens, for example, if the other party does not reply, or only replies with grunts when more participation would be expected. Then the bond between the parties is broken and re-initiating the communication anew will require more effort.

In technology-mediated interaction, e.g. with email or text messaging, the possibility for breakdowns is inherently present all of the time, as the medium allows only for discontinuous messaging, not an ongoing two-way interaction. Problems arise easily for instance if a question is not replied or receiving the

\footnotetext{
(C) The Author 2008
}

Published by the British Computer Society response takes longer than is seen as appropriate.

When communication flows smoothly, messages are exchanged and replied in due time with understandable delays in between. This kind of reciprocity in communication can also be seen as a form of gift-giving, and this approach has already been successfully applied in some CSCW-oriented publications on mobile messaging $[11,12]$. These studies have shown that interpreting mobile messaging as gift-giving not only manages to capture some of the essence of messaging itself, but also a larger context of practices related to displaying and maintaining friendship. For instance, a gift in a form of a message can be reciprocated by letting the sender borrow one's own phone for a while, thereby letting the sender see one's personal content on a mobile phone and make calls on the other's expense [12].

While the previous studies have emphasized the positive aspects of gifting, this paper addresses situations in which gifting becomes problematic in messaging-based communication. The examples are from a field study of a mobile comic strip creator Comeks $^{1}$ [7]. While the majority of messaging with Comeks provided happy experiences for the users, a small percentage of exchanges contained aspects of the negative side of gift-giving. By presenting and analyzing these situations in this paper, we attempt to develop design implications to alleviate similar problems in other systems.

\section{RESEARCH ON GIFT-GIVING}

The interpretive framework of gift-giving originates from one of the seminal anthropological works of the $20^{\text {th }}$ century, from an essay by Marcel Mauss (1872-1950) [4]. Mauss analyzed societies as structured systems of gift exchange, and used examples from archaic cultures in Polynesia and Melanesia of the Pacific and Indians in northwestern America to illustrate this point. He maintained that gifting is one of the fundamental unifying processes in a society, promotes positive interdependence and enables mutual trust and lasting relationships. Every member of the community participated in this ongoing process, and the exchanges permeated all parts of social life.

Especially relevant to this paper, Mauss identified three obligations related to gift-giving: obligation to give, accept and reciprocate. First of all, every member of a society was compelled to offer gifts. Refusing to do that would have been a sign of rejecting the mutual bond of alliance. The same applied to rejecting a gift when it was offered, or refusing to pay it back. If not returned, the donor was seen as having a hold of the receiver - a situation that put in danger that person's autonomy.

These ideas about gift-giving can be applied also to exchanges of more intangible goods. Kollock [3] has applied the frame-

\footnotetext{
${ }^{1}$ This paper concerns the previous version of Comeks. The newer version has already addressed the problems presented in this study. For more about Comeks, see www.comeks.com
} 
work to describe processes in online Linux developer communities, focusing on motivations for giving programming tips for public use. The system of gift-giving appears to hold although posting content to a forum for anonymous others is a case in which reciprocal exchanges are not realized as directly as in personal interaction. Skågeby [9] has analyzed photo-sharing contributions in online photo-sharing system Flickr. Users appear to have concerns about who is able to see their content, or "receive the gifts". This is because photos in Flickr can spread to other digital spheres (blogs, RSS feeds, email circulation) in uncontrolled ways. To avoid this, users tend to use alternative distribution channels (e.g. upload to a personal website and email notification thereafter) at certain occasions.

Most relevant to this paper is the work carried out by Taylor and Harper $[11,12]$. Using observations and group interviews, they studied the use of mobile phones among teenagers in an English high school (sixth form). Mobile phones were an integral part of teenagers' social life, and also a medium for gift exchange - not only in the form of phone call and text message exchanges, but also as permissions for certain others to make calls with one's phone and see its personal content.

In this process, sending a message or lending the phone represents the mutual trust that must be shown to one's friends. The obligation to accept is related to reading the incoming message in due time and reacting to it if needed, thus showing that the donor's role in the social context is acknowledged. Finally, people are obliged to reciprocate in order to show to the other party that the relationship is valued. It also counterbalances the hierarchical shift caused by the previous gift.

Taylor and Harper show how these rules become evident in the interviews, expressed as users' own descriptions about their phone use habits. In the following, after a brief presentation of the messaging software, we will see how the rules were rendered visible when the reciprocity could not be maintained.

\section{COMEKS USER TRIAL}

Comeks is a mobile comic strip creator, designed to provide camera phone users with a richer variety of expressions than allowed by the normal multimedia messages (MMSs). While a normal MMS lets user send images and pieces of text on different slides of a message, Comeks also helps the user to turn images into narratives annotated with comic-style elements such as speech bubbles. Images can also be rotated, resized and toned in a variety of ways to increase their expressiveness.

4 boys and 4 girls (aged 17 to 18 years) from an urban high school in Helsinki, Finland were recruited to the user trial. Members already knew each other before the trial started, being friends with each other. The trial lasted 9 weeks. A detailed description of the field study has been published in [7].

Two kinds of primary data were gathered in the study. One was the multimedia messages sent and received (both Comeks and normal MMSs). This traffic was collected unobtrusively with special logging software [4]. The logs were sent every night to a server and were thus observable almost in real time.

The other primary data consisted of interviews in which the users described the situations, motivations and reactions related to each sending and receiving event. The interviews were held with one user at a time, at approximately three-week intervals, to collect fresh memories about each event. Printouts of the messages were used in these discussions.

The study followed the principles of qualitative research (see e.g., [8]), and focused on understanding emerging phenomena rather than quantifying and categorizing the answers. During the study, gift-giving emerged as such a phenomenon in the communication practice. Users occasionally remarked how they sometimes felt negatively upon receiving or creating messages. Because of this, additional questions about gift-giving were inserted into the subsequent interviews.

\section{GIFT-GIVING PROBLEMS}

By looking at the content sent and the interview data, it became apparent that while some messages were created because of utility-oriented reasons, most of them were created for fun. Either the user experimented with Comeks out of sheer joy of seeing how the different editing features affected the images, or just to send something to the others and make them happy.

Altogether, 66 excerpts were found in the interviews touching the issues of gift-giving. We will present them organized according to the three obligations, highlighting the constraints that hindered gift-giving in mobile multimedia messaging.

Problems in gift-giving can also be noticed by looking at the numbers of messages. In total, participants sent 118 multimedia messages in 9 weeks. These messages constituted 96 conversations of which only $11(11 \%)$ had more than one message. Given the figures obtainable from text messaging studies that report percentages as high as $49 \%$ [1] (see also [10]), this can be taken as a sign of relatively infrequent turn-taking.

\subsection{Obligations to give}

With respect to mobile messaging with Comeks, obligation to give was not a big issue to the users in that they did not feel obliged to be actively sending messages to each other. Only a very few remarks were made about those users who were not communicating actively with Comeks. What is more important is that in many cases when a message was sent, the sender was expecting a reply even though his or her message was not an inquiry. Here are two quotes from Eeva related to the two messages shown in Figure 1 illustrating this:

Eeva, on message A: Always when we have a party there comes a moment with a feeling that now funky! For those moments, Asif sometimes makes a minidisc or burns a CD [...]I sent this to Asif right away, like "wow!" since the time I saw the present I thought, how has someone guessed to give such a great present. [...] Also to this [message] I would've liked that Asif replies back that "yeah yeah, let's play that next time we have a party". Or I don't remember if he sent me anything back actually.

Eeva, on message B: When I sent a message about that Las Vegas program with the actor's face, I was thinking, please reply anything. Just about anything would have been fine.

However, the recipient did not always understand that a reply was expected - that a gift was meant to be returned. In contrast to Mauss, it seems that the users understood the rules of gift
A

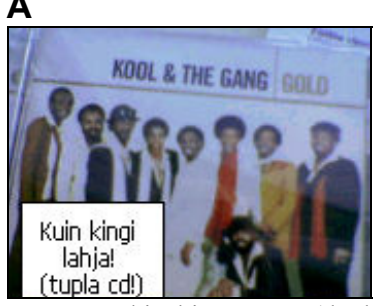

"How cool is this present! (double cd!)"

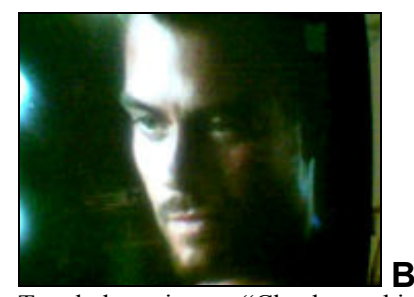

Text below picture: "Check out this total hottie on Las Vegas. On Channel 4 ;) *mother may I* wink wink"
Figure 1. Two messages to which the sender was expecting replies: a message about a Christmas gift (A) and a reminder that a favourite TV show is now on TV (B). 
exchange in different ways, thus unintentionally causing bad feelings to each other. The recipients' comments to the same messages shown in Figure 1 show this:

Asif, on message A: Yeah she just sent this one to me. $A C D$ with all the Kool and the Gang's best songs, a double CD. But to that I didn't send anything back. I however went to net and wrote to her, she was there on the wire.

Tina, on message B: That was really a great one, I had been reading for exams the whole evening, started to feel really drained. Also that one was one of those that you don't need to answer, but it was still a message that I'd reply anyway, and I also did so. If someone remembers you without any need to do so then it's nice to throw some stuff back as well.

Thus, although Asif had acknowledged Eeva's message in a chat session, Eeva did not perceive that as an adequate return gift. On the other hand, Tina replied back to Eeva with a Comeks message, but said it would have not been necessary.

\subsection{Obligation to accept}

In other cases, the sender really did not mean that the message would need to be replied, but the recipient felt an obligation to do so. This resulted sometimes in a wish that the message was not sent at all in the first place. This had often to do with "unrelatedness": a message had arrived unanticipated, without any hint on what it is related to or how to answer to it:

Asif: About this message I don't know, I think I got it the day when I was reading for the math exam. I don't how this relates... He just sent this to me.

Asif: I just looked at the message and thought "oh well, okay" and put the phone away. I wondered what was the point in it.

Tina: This was the first message of all of these that I thought like... I thought that this should continue somehow because I couldn't figure out the point of this single frame. That if it would continue then there would be some story. Then it could have... This was a message that I wondered where is the point.

Asif: First I wondered why is he sending a message like this to me? I was like "ok, fine...This is funny and created well, but ok, yeah, if you have something to tell, then,..."

The users therefore sometimes felt uneasy about the obligation to accept a gift. This feeling was possibly emphasized due to two factors: Firstly, by the way mobile messaging works, the sender has power over the recipient in that the recipient cannot control the messages that arrive in the handset. The recipient therefore must accept the gift or delete it without looking at it. The other reason is related to the experiences accumulated about the gift-giving ritual and failures to reciprocate to previous messages. This obligation we will look at next.

\subsection{Obligation to reciprocate}

One problem related to reciprocating a gift was already presented above - that of not understanding a requirement to reciprocate in the same way. Yet other reasons could also be identified. The instant nature of mobile messaging imposes a need to reply quickly, which runs against the possibility of finding material for a comic strip. Therefore the users expressed on many occasions that they would have liked to reply but found nothing to photograph. Another problem also related to the need for quick replies was the issue of inspiration. Creating a comic strip is a creative undertaking, and strips with satisfying storylines and visual designs cannot always be achieved. A third problem was that the recipient often was in the middle of doing something and had no time to construct an answer. At a later time, when the user would have had time, he or she could already feel awkward about sending a reply to such an old message. Therefore sometimes no replies were sent at all. These problems can be found in the following excerpts:

Anne: If you want to create a really nice strip you need to have time to think about it. It's not about the actual writing of the message, Comeks's really fast in it. But the thinking about what you want to send. So when you sit in a bus you have time for that.

Asif: If you are sitting at home on weekend, looking a bit rotten, it's not that fun to start sending pictures to another person.

Asif: If you receive a message when you're walking in the city centre, then replying is like taking a photo of a something boring like a tree and saying "ha, haa, your message was cool, here's mine".

Because of the above reasons it sometimes turned out to be impossible to return a gift of a comparable value. A gift smaller than the one received was not seen as proper conduct.

Anne: I intended to send some message back, a thank you message or so, because I liked that strip a lot. But then I did not know how to meet the same level of quality.

Tea: If you have put some effort [into creating the message] then the other party should do that as well. If I send a picture message and ask are we going out tonight together, and the other one replies just "OK", it's boring.

\subsection{Resulting messaging strategies}

As a result of the obligations being perceived as difficult in all the three categories, users developed strategies and attitudes that helped them to cope with the feelings arising out of breaking social norms. Message recipients adopted sometimes a "I don't care, this is not for me" attitude, apparent in the statements by Asif in Section 4.2. Another strategy was to create a message once in a while that would make up all the debts of obligations that had been piled on the user. It made the user feel better although the contents in those messages often did not represent anything of great value to the sender.

For active Comeks users, the situation appeared in a different light. In order not to remain constantly irritated by getting no feedback to messages, two different strategies could be adopted. One was to start tailoring the messages more carefully with the recipient in mind, this way making them easier to reply to. Eeva, the most active user in the study, expressed this as follows in an interview when talking about a humorous collage of portraits of three other users she had sent:

At least I always thought when I was creating them, I thought what would I reply back if I received something like this [...] That's why here I wanted to have the boys in the strip and I took a photo of all of them.

Another strategy was to develop a more relaxed attitude towards messaging, and create messages that did not contain a hint of an expected reply. This was the strategy adopted by Tina. For instance, she used Comeks to thank her friends for a good time in a bar the previous night. She was also happy that she did not probably cause hard feelings in the receiving end.

\section{Discussion}

As Comeks was a new medium for all of its users in the study, completely new norms of interaction could have been developed around it - including ones that were free of reciprocal 
obligations of gift-giving. However, the data presented in this paper shows that messaging with Comeks reflected the same kind of social norms of gift-giving as can be seen in other areas of social life.

Even when encapsulated within the normativity of gift-giving, it is interesting that these rules in the above cases worked against enjoyable conversation while they could have equally likely strengthened the social relationships within the group and contributed to a cycle of increased communication.

The fact that messages can become too laborious to reciprocate is an issue that may severely hinder the uptake and appropriation of a new communication technology. It must be emphasized again that the problems discussed here represent only a sample of Comeks data, and in other parts of the interviews the users were talking about their conversation in a more positive light (see [7]). Nevertheless, the findings still warrant the following design implications for messaging system design:

- Decreasing the effort to create long messages. In the study, long strips with storylines were the most appreciated of all messages. Their creation should therefore be made as effortless as possible e.g. with optional templates for message creation. Such lightweight tools for increasing message fidelity could also be useful in other systems similar to Comeks.

- Group-wide messaging. Messages sent to many people should be replyable to all parties as well. This would invite more discussion and provide a forum for showing respect towards the sender.

- Support gift-giving on other than a message-per-message basis. Many problems in the study stemmed from users' expectations that a comic strip should be reciprocated with another strip. A better way could have possibly been a multistaged reply: to deliver a quick acknowledgment immediately and a longer answer later, possibly through another media channel. This would provide better first-hand feedback to the sender and a more convenient way for the recipient to reply.

- Reciprocating with gifts of a different kind. The messaging medium should be embedded into the larger social system of gift exchange and not treated as an "island". For instance, a message could be reciprocated with a piece of a different kind of digital content, such as an email or a link to a video in the internet. Integration with other systems - a topic that extends the previous point - would help in achieving this.

- Support the negotiation of gift-giving. Examples showed that the principles of gift-giving were not equally understood across all users. While not a simple goal to achieve, supporting negotiation of these principles through and around the medium is a topic to keep in mind during design.

Although these implications have not been explicated before this paper, the improvements in the new version of Comeks are much in line with them, especially through the support of communicating through multiple electronic channels and provision of optional strip templates and software versions of different fidelity. With respect to messaging systems in general, we are working on developing these ideas into more concrete implications for design.

\section{ACKNOWLEDGMENTS}

The author wants to thank the people at Comeks for cooperation and Mark Perry for comments. This work was supported by Mobile Content Communities project funded by Finnish Funding Agency of Technology, Academy of Finland, and the Graduate School of User-Centred Information Technology.

\section{REFERENCES}

[1] Grinter, R.E. and Eldridge, M. Wan2tlk?: Everyday text messaging. In Proc. CHI2003. ACM Press, New York, NY, 2007, 441-448.

[2] Heritage, J. Garfinkel and Ethnomethodology. Polity Press, New York, NY, 1984.

[3] Kollock, P. The economies of online cooperation: gifts and public goods in cyberspace. In Smith, M. A. and Kollock, P. (eds.). Communities in Cyberspace. Routledge, London, UK, 1999, 220-239.

[4] Mauss, M. The Gift: The Form and Reason for Exchange in Archaic Societies. Routledge, London, 1950/2004.

[5] Raento, M., Oulasvirta, A., Petit, R., and Toivonen, H. ContextPhone: A prototyping platform for context-aware mobile applications. IEEE Pervasive Computing 4, 2 (2005), 51-59.

[6] Sacks, H., Schegloff, E.A., and Jefferson, G. A simplest systematics for the organization of turn taking in conversation. Language 50, 4 (1974), 696-735.

[7] Salovaara, A. Appropriation of a MMS-based comic creator: From system functionalities to resources for action. In Proc. CHI2007. ACM Press, New York, NY, 2007, 1117-1126.

[8] Silverman, D. Interpreting Qualitative Data: Methods for Analysing Talk, Text and Interaction. SAGE, London, 1993.

[9] Skågeby, J. Semi-public end-user content contributions A case-study of concerns and intentions in online photosharing. International Journal of Human-Computer Studies, 66, 4 (2008), 287-300.

[10] Spagnolli, A. and Gamberini, L. Interacting via SMS: Practices of social closeness and reciprocation. British Journal of Social Psychology 46, 2, 343-364.

[11] Taylor, A.S., and Harper, R. Age-old practices in the 'new world': a study of gift-giving between teenage mobile phone users. In Proc. CHI 2002. ACM Press, New York, NY, 2002, 439-446.

[12] Taylor, A.S., and Harper, R. The gift of the gab?: A design oriented sociology of young people's use of mobiles. Computer Supported Cooperative Work 12, 3 (2003), 267296. 